\title{
Influence of the Supramolecular Organization on the
}

\section{Magnetic Properties of Poly(3-alkylthiophene)s in Their}

\section{Neutral State.}

Steven Vandeleene ${ }^{a, d}$, Mihaela Jivanescu ${ }^{b, d}$, André Stesmans ${ }^{b, d}$, Jo Cuppens ${ }^{c, d}$, Margriet J. Van Bael ${ }^{c, d}$, Thierry Verbiest ${ }^{a, d}$, and Guy Koeckelberghs ${ }^{a, d *}$

${ }^{a}$ Laboratory of Molecular Electronics and Photonics, Katholieke Universiteit Leuven, Celestijnenlaan 200F, B-3001 Heverlee, Belgium

${ }^{\mathrm{b}}$ Semiconductor Physics Laboratory, Katholieke Universiteit Leuven, Celestijnenlaan 200D, B-3001 Leuven, Belgium

${ }^{\mathrm{c}}$ Laboratory of Solid State Physics and Magnetism, Katholieke Universiteit Leuven, Celestijnenlaan 200D, B-3001 Leuven, Belgium

${ }^{\mathrm{d}}$ INPAC -Institute for Nanoscale Physics and Chemistry, University of Leuven, Belgium

guy.koeckelberghs@chem.kuleuven.be

RECEIVED DATE (to be automatically inserted after your manuscript is accepted if required according to the journal that you are submitting your paper to)

Abstract. The influence of the supramolecular behavior on the magnetic properties of head-to-tail coupled poly(3-alkylthiophene)s (P3AT) is reported. Samples with a different molar mass were 
prepared and differences in their behavior were illustrated by UV-vis and CD spectroscopy. In addition, tuning of the supramolecular organization was also accomplished by applying thermal annealing. ESR spectroscopy was performed on powder samples. From the line features, information on the nature of the unpaired spins, the mobility and the supramolecular order of the sample was derived. These spins are paramagnetic. SQUID magnetometry, in contrast, shows for all samples a ferromagnetic behavior at $5 \mathrm{~K}$ and a superparamagnetism at $300 \mathrm{~K}$. The coercivity does not depend on the degree of supramolecular order, but is inherent to the molecular structure, and related to this, the $\pi$-interactions. The saturation magnetization, on the contrary, seems to be mainly influenced by the fraction of planarized polymer chains.

Keywords. Conjugated polymers, magnetism, ESR, SQUID, poly(3-alkylthiophene).

\section{Introduction}

For almost three decades, $\pi$-conjugated polymers have been explored extensively. Besides their optical, electrical and electronic properties ${ }^{1}$ also the magnetic behavior has been investigated, albeit to a lesser extent. In order for an organic compound to exhibit ferromagnetism, unpaired electrons (spins) should be present. Mostly, spins are intrinsic ${ }^{2-4}$ to the material or introduced as radicals, carbenes or by oxidation. ${ }^{2-3,5}$ A second requirement is a pathway by which unpaired spins can mutually interact (spin coupling). ${ }^{6}$ In this point of view, a conjugated $\pi$-system is ideal to act as a (ferro)magnetic linker between different spins. However, the substitution pattern and the connectivity are crucial parameters to have ferromagnetic spin coupling, aligning spins in the same direction of an external magnetic field. ${ }^{7}$ Quite some "high-spin" molecules ${ }^{8-9}$, oligomers ${ }^{10}$ and polymers ${ }^{11-14}$ have been reported based on this approach and remarkable advances have been achieved.

Besides the "high-spin" molecules, also some typical conjugated polymers were reported, showing magnetic behavior under very specific conditions. Typical examples are poly(aniline)s ${ }^{15-18}$, poly(pyrrole) $)^{19}$, regio-irregular poly(3-alkylthiophene) $\mathrm{s}^{20-24}$ and (substituted) poly(acetylene)s. ${ }^{25}$ In all these examples, the materials were chemically or electrochemically doped, introducing spins (polarons). 
In contrast to these results, we have previously reported on the magnetic behavior of neutral (undoped) poly(thiophene)s carrying an alkyl, alkoxy or alkylthio sidechain. ${ }^{26}$ A comparison of the ESR and SQUID data reveal the presence of (at least) two spin systems. A first electron spin system gives rise to a paramagnetic behavior. A second spin system (of which the origin is unknown), results in a superparamagnetic behavior at $300 \mathrm{~K}$ and (for most polymers) a ferromagnetic behavior at $5 \mathrm{~K}$. The ESR-active system is not responsible for the ferromagnetic behavior at $5 \mathrm{~K}$. This has been concluded from the fact that it exhibits a paramagnetic behavior and that there is a large discrepancy between the number of spins $\left(\mathrm{S}=\frac{1}{2}\right)$, determined by ESR, and the number of Bohr magnetons (magnetic moment of an electron) measured by SQUID magnetometry. Concerning the presence of ferromagnetism and the coercivity, it was found that polymers in which strong $\pi$-interactions between stacked polymer chains are present, show the highest coercivity. Therefore, it can be hypothesized that these $\pi$-interactions determine the ferromagnetic behavior (coercivity).

In this article, the influence of supramolecular organization and $\pi$-stacking of a chiral substituted, head-to-tail coupled poly(3-alkylthiophene) (HT-P3AT) on its magnetic behavior is investigated. First, since aggregate formation is influenced by the molar mass of these polymers, samples with different molar masses were prepared by fractionation. Differences in their supramolecular behavior are probed by UV-vis and circular dichroism (CD) spectroscopy, while the magnetic properties are evaluated by ESR spectroscopy and SQUID magnetometry. A chiral rather than an achiral substituent was preferred, since this allows the use of powerful chiroptical techniques which can provide important information on the supramolecular structure of the material. Second, supramolecular organization and resulting changes in the $\pi$-interactions were manipulated by thermal annealing ${ }^{27}$ and the magnetic response was again investigated. 


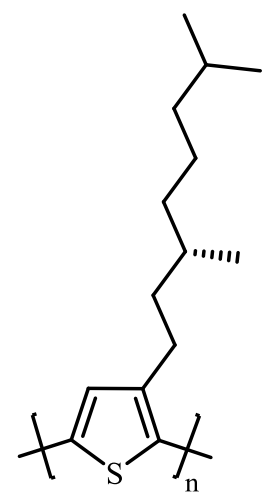

HT-P3AT

$\overline{\mathrm{M}}_{\mathrm{n}}=7.0 \mathrm{~kg} / \mathrm{mol} \quad \mathbf{P 1} \quad \overline{\mathrm{M}}_{\mathrm{n}}=12.5 \mathrm{~kg} / \mathrm{mol} \quad \mathbf{P 3}$

$\overline{\mathrm{M}}_{\mathrm{n}}=10.3 \mathrm{~kg} / \mathrm{mol} \quad \mathbf{P 2} \quad \overline{\mathrm{M}}_{\mathrm{n}}=16.0 \mathrm{~kg} / \mathrm{mol} \quad \mathbf{P} 4$

Figure 1. Structure of the chiral HT-P3ATs P1-4.

\section{Materials and Methods}

Gel permeation chromatography (GPC) measurements were done with a Shimadzu 10A apparatus with a tunable absorbance detector and a differential refractometer in tetrahydrofuran (THF) as eluent toward polystyrene standards.

UV-vis and CD spectra were recorded with a Varian Cary 400 and a JASCO 62 DS apparatus respectively. The DSC experiments were performed on a DSC 7 from Perkin Elmer

Films were prepared by spincoating from chloroform solutions on a glass substrate. To vary the film thickness, polymer solutions with different concentrations $(9.4 \mathrm{mg} / \mathrm{mL}, 24 \mathrm{mg} / \mathrm{mL}$ and $40 \mathrm{mg} / \mathrm{mL})$ were used and the preparation parameters were kept constant (spinning speed: $1500 \mathrm{rpm}$; spinning time: $30 \mathrm{~s}$ ). A Mettler-Toledo FP900 Thermosystem was used for sample annealing. Polymer films were heated at $170^{\circ} \mathrm{C}$ for several minutes. Afterwards, fast cooling was obtained by dipping the film in methanol, cooled at $-78^{\circ} \mathrm{C}$ and dried under an air flow. Slow cooling was accomplished at a cooling rate of $10^{\circ} \mathrm{C} / \mathrm{min}$. Powder samples, washed with an alcoholic hydrazine solution and methanol, respectively, were annealed (after drying) between two Teflon layers at $170^{\circ} \mathrm{C}$ for several minutes. Fast cooling was obtained by dipping the Teflon layer in methanol, cooled at $-78^{\circ} \mathrm{C}$ and dried under an air flow. Slow cooling of the powder samples was accomplished at a cooling rate of $-10^{\circ} \mathrm{C} / \mathrm{min}$. After thermal treatment, the powder samples were gently removed from the Teflon substrate. 
The powders were weighted and stored under inert (argon) atmosphere. It should be stressed that Soxhlet extraction in essence delivers nickel-free polymer samples as previously motivated. ${ }^{26}$

Conventional continuous slow-passage X-band ( $~ 9.2 \mathrm{GHz})$ ESR measurements were carried out at room temperature using a Jeol FA100 spectrometer, where low microwave power $\left(\mathrm{P}_{\mu} \leq 1 \mathrm{~mW}\right)$ first derivative-absorption $\mathrm{dP}_{\mu} / \mathrm{dB}$ spectra were recorded through applying sinusoidal modulation $(\sim 100 \mathrm{kHz}$; amplitude $B_{m} \sim 0.35 \mathrm{G}$ ) of the externally applied magnetic field B. Some observations were made in a mode-built K-band $(\sim 20.6 \mathrm{GHz})$ setup, as described elsewhere. ${ }^{28}$ A co-mounted calibrated $\mathrm{MgO}: \mathrm{Mn}^{2+}$ reference sample was used for absolute $\mathrm{g}$ factor and spin density ( $\operatorname{spin} \mathrm{S}=\frac{1}{2}$ ), calibration, with the latter performed through double numerical integration of the detected $\mathrm{dP}_{\mu} / \mathrm{dB}$ spectra. At $\mathrm{X}$ band, the $\mathrm{g}$ values of the $3^{\text {rd }}$ and $4^{\text {th }}$ lines of the $\mathrm{Mn}^{2+}\left({ }^{55} \mathrm{Mn} ; 100 \%\right.$ natural abundance; nuclear spin $\left.\mathrm{I}=5 / 2\right)$ sextet were calibrated as $2.0338 \pm 0.0001$ and $1.9807 \pm 0.0001$, respectively. The attained absolute and relative accuracy is estimated at $\sim 20 \%$ and $\sim 5 \%$, respectively. Signal averaging (typically $\sim 50$ scans) was routinely applied to enhance spectral quality.

SQUID-based magnetometry is carried out in a MPMS-XL magnetometer (Quantum Design). The powders are weighted and a typical amount of $15 \mathrm{mg}$ is fixed between small pieces of pure cotton wool inside the non-magnetic plastic transparent sample tube. No capsules are used to hold the powder in order to avoid any magnetic contributions from the capsules interfering with the measured signal. The magnetic signal from the plastic sample tube with cotton wool (without the powder sample) is measured separately and shows a small diamagnetic contribution which is linear in applied field and can therefore be distinguished from non-linear contributions from the powders. Magnetization was measured at different fixed temperatures as a function of magnetic field in RSO (reciprocating sample option) operation mode.

When measuring weak magnetic responses, one needs to be very cautious for (magnetic) contaminations, Therefore, working conditions were kept as clean as possible and any contact of the sample powders with metals (spatula, ...) was avoided. Moreover, control ESR experiments were 
performed on several samples before and after the SQUID measurements . No traces of ferromagnetic metal $(\mathrm{Fe}, \mathrm{Ni}, \ldots)$ contaminations could be detected in the ESR spectra.

\section{Results and Discussion}

\section{Materials}

Regioregular, head-to-tail poly(3-alkylthiophene), substituted with a chiral alkyl side chain, was prepared by the Grignard Metathesis procedure, described by McCullough and coworkers. ${ }^{29}$ After polymerization, the crude polymer, having a polydispersity of 2.25 , was precipitated in methanol, isolated and fractionated.

The fractionation ${ }^{30-33}$ of the crude polymer was accomplished by means of a Soxhlet extraction with different solvents. First, methanol and acetone were used to remove catalyst traces, unreacted monomers and oligomers. Second, the polymer was extracted with $n$-pentane (P1), $n$-hexane (P2), dichloromethane (P3) and chloroform (P4). Finally, all isolated polymer fractions were concentrated, precipitated in icecooled methanol, filtrated and dried in vacuo until a constant mass was reached. The number averaged molar mass $\left(\overline{\mathrm{M}_{\mathrm{n}}}\right)$ and polydispersity $(\mathrm{D})$ of the isolated fractions were determined by GPC (GPC traces, see S1) and are summarized in Table 1. The samples show a significant difference in molar mass and in all cases, the polydispersity is below 1.3. The degree of polymerization was also calculated by relative integration of the inner and terminal methylene ${ }^{1} \mathrm{H}$ NMR resonances. 
Table 1. Molar mass and DSC data of P1-4.

\begin{tabular}{lllllll}
\hline Polymer & $\begin{array}{l}\overline{\mathrm{M}}_{\mathrm{n}}{ }^{\mathrm{a}} \\
(\mathrm{kg} / \mathrm{mol})\end{array}$ & $\mathrm{D}^{\mathrm{b}}$ & ${\overline{\mathrm{X}_{\mathrm{n}}}}^{\mathrm{c}}$ & ${\overline{\mathrm{X}_{\mathrm{n}}}}^{\mathrm{d}}$ & $\begin{array}{l}\mathrm{T}_{\mathrm{m}}{ }^{\mathrm{e}} \\
\left({ }^{\circ} \mathrm{C}\right)\end{array}$ & $\begin{array}{l}\Delta \mathrm{H}_{\mathrm{m}}{ }^{\mathrm{e}} \\
(\mathrm{J} / \mathrm{g})\end{array}$ \\
\hline P1 & 7.0 & 1.2 & 32 & 17 & 103 & 8.7 \\
P2 & 10.3 & 1.3 & 46 & 20 & 121 & 11.4 \\
P3 & 12.5 & 1.2 & 56 & 28 & 131 & 16.1 \\
P4 & 16.0 & 1.1 & 72 & 34 & 137 & 16.5
\end{tabular}

${ }^{\mathrm{a}}$ Number averaged molar mass, ${ }^{\mathrm{b}}$ Polydispersity and d degree of polymerization, Determined by GPC in THF, towards polystyrene standards. ${ }^{\mathrm{d}}$ Degree of polymerization, determined by relative integration of the inner and terminal methylene ${ }^{1} \mathrm{H}$ NMR resonances. ${ }^{\mathrm{e}}$ Measured by $\mathrm{DSC}$ with a heating rate of $20^{\circ} \mathrm{C} / \mathrm{min}$; the second heating run was used.

\section{Chiroptical behavior: Molar mass dependency}

The supramolecular organization and $\pi$-stacking were investigated by UV-vis and CD spectroscopy. As ESR and SQUID measurements are typically performed on polymer powders, prepared by precipitation from methanol, a good approach to characterize differences between the polymer powders is by comparing the chiroptical behavior of P1-4 in their aggregated form in solution.

The chiroptical behavior of P1-4 was investigated both in good solvent and upon addition of nonsolvent. In good solvents, such as chloroform, the $\lambda_{\max }$ of P1-4 is more or less equal (See S3), indicating that the conjugation length of the actual "chromophore" is not limited by the degree of polymerization. However, when the solvent quality diminishes upon addition of methanol, aggregation is induced. In all cases, the UV-vis spectra (Figure 2, a) show a red-shift of the $\lambda_{\max }$, which is more pronounced for higher molar masses. In fact, the samples are composed of three "chromophores". One band ( 450 nm) arises from disordered, coiled polymer chains. A second transition $(\sim 530 \mathrm{~nm}$, with vibronic fine-structure $)$ is localized on planar, stacked chains. Finally, all samples show a red-shifted shoulder at around $615 \mathrm{~nm}$. This distinct, low-energy absorption band can be attributed to a transition dipole moment, delocalized 
over multiple, aggregated polymer chains. ${ }^{34-35}$ This phenomenon is, however, not limited to HT-P3AT ${ }^{36}$. Quite some semicrystalline, $\pi$-conjugated polymers show this very specific absorption band as well. ${ }^{37-38}$ The occurrence of this aggregation-related absorption band is also visible in the CD spectrum (Figure 2, b). While the first UV-vis transition is CD silent, the others give rise to Cotton effects. A bisignate Cotton effect, due to chiral exciton coupling, is associated with the planarized polymer chains. Also a monosignate Cotton effect, corresponding to the red-shifted transition, is present, caused by a helical oriented transition dipole moment, delocalized over multiple polymer chains. The four polymer samples essentially differ in the relative ratio of the aggregation-related absorption band. This can already be observed by the more pronounced shoulder at $615 \mathrm{~nm}$ for $\mathbf{P 4}$, compared to P1. Also the $\mathrm{g}_{\text {abs }}$-value, $(=$ $\Delta \varepsilon / \varepsilon$ ) which is a measure for the chirality of a system, was calculated (Figure 2, c) and shows a maximum value of $7.5 \times 10^{-3}$ for $\mathbf{P 4}$. Therefore, already as first evidence, UV-vis and CD spectra confirm that a higher crystalline fraction, in which the polymer strands are chirally stacked and mutually interact, is present in high molar mass polymers. 

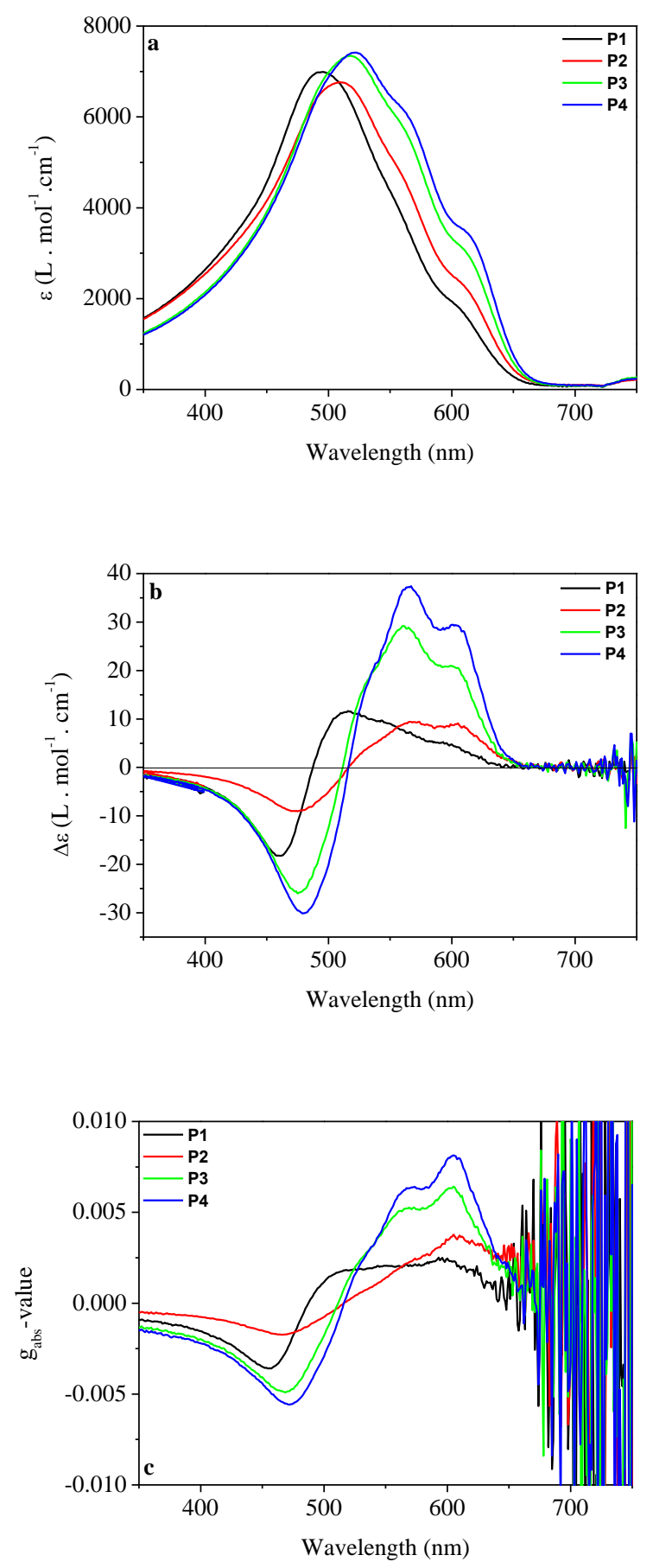

Figure 2. UV-vis spectra (a), CD spectra (b) and $\mathrm{g}_{\mathrm{abs}}$-value (c) of P1-4 in a mixture of chloroform and methanol (2/8).

In order to quantify the aggregation-related absorption band, the different UV-vis spectra were deconvoluted as shown for P4 in Figure 3 (See also S4) using a linear programming method. ${ }^{39}$ Figure $3 b$ shows the relative, integrated absorption of the red-shift band as a function of the molar mass of the 
polymer samples. It is clear that a more intense aggregation-related absorption band correlates with higher molar masses. This observation complies with the observations made by other groups, using Xray diffraction (XRD) and differential scanning calorimetry (DSC) on polymer samples having different molar masses. ${ }^{40-42}$ High molar mass HT-P3AT has a higher crystalline fraction compared to lowmolecular HT-P3AT, ${ }^{40,43}$ which is again confirmed by our results. As a consequence, the red-shifted absorption, for which $\pi$-interactions play a crucial role, is more pronounced in HT-P3ATs with high molar masses, as they shows a higher degree of order.
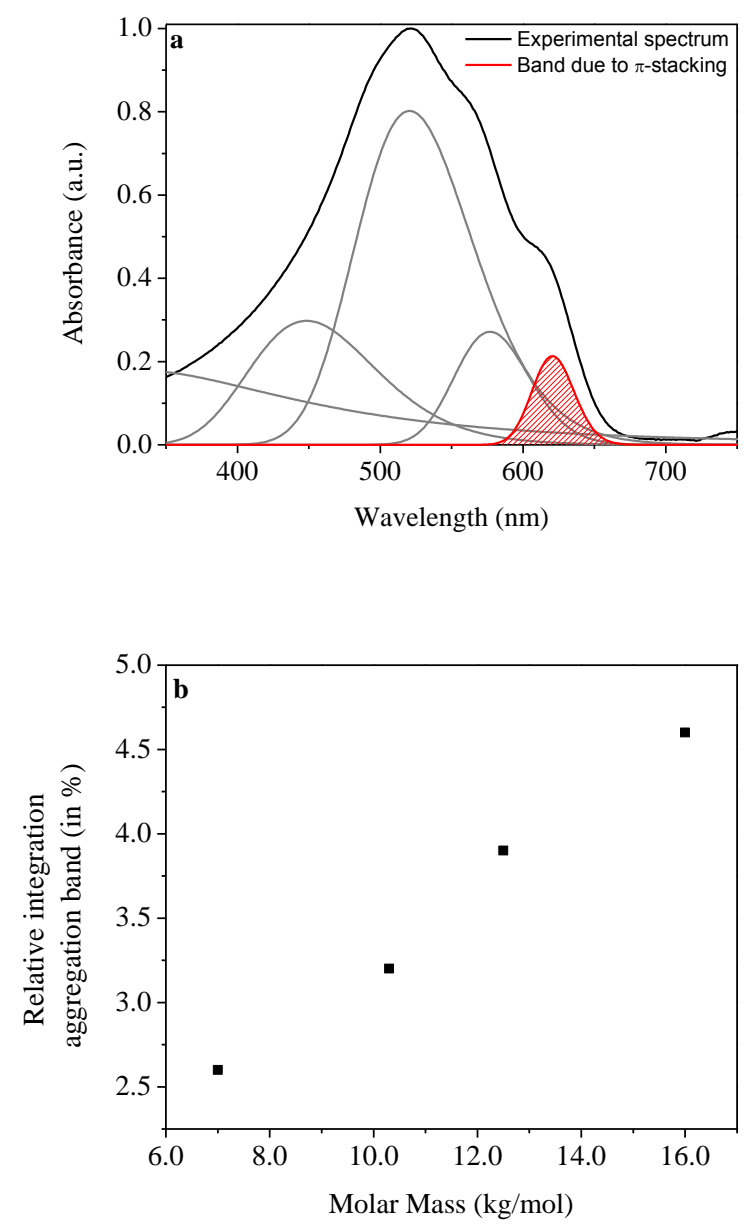

Figure 3. (a) Deconvolution of the UV-vis spectra, resolving the aggregation-related absorption band, shown for P4; (b) Relative integration of the aggregation-related absorption band (shaded peak) as function of the molar mass, determined by GPC. 
In order to verify that the higher-molar mass samples indeed have a higher degree of crystallinity, we performed some DSC experiments. All polymers are semi-crystalline and show similar thermograms, suggesting that the same type of crystals is formed. This is in line with the fact that the molar mass does not change dramatically and remains in the region where chain-extended crystals are expected. ${ }^{44-45}$ The melting temperature $T_{m}$ and melting enthalpy $\left(\Delta \mathrm{H}_{\mathrm{m}}\right)$ were typically measured at the second heating run and are shown in Table 1. A similar behavior as found for poly(3-hexylthiophene) is present: both $\mathrm{T}_{\mathrm{m}}$ and $\Delta \mathrm{H}_{\mathrm{m}}$ increase with $\overline{\mathrm{M}_{\mathrm{n}}}$. Since $\Delta \mathrm{H}_{\mathrm{m}}$ increases with increasing degree of crystallinity, it can be concluded that the degree of crystallinity indeed increases with increasing $\overline{\mathrm{M}_{\mathrm{n}}}$.

\section{Chiroptical behavior: Influence of annealing}

Not only the molar mass influences the aggregate formation. Also thermal treatment of the polymer samples (annealing) has a significant influence on the amount, the shape and the size of aggregates. To study the effect of annealing on the magnetic properties of HT-P3AT, several powder samples of P3 were subjected to different heat treatments. The powders were gently pressed $\left(1 \mathrm{ton} / \mathrm{cm}^{2}\right)$ to ensure a

good thermal contact. Pressure has shown to influence the supramolecular structure of P3ATs as well. ${ }^{46}$ However, the pressure required is several orders of magnitude higher. Here, we "press" the powder to ensure good thermal contact for the thermal annealing. In order to monitor the changes on a supramolecular level, films of $\mathbf{P 3}$ were subjected to the same thermal treatment as the powders and were characterized by UV-vis and CD spectroscopy.

A film of $\mathbf{P 3}$ was spincoated $(1500 \mathrm{rpm})$ from a chloroform solution $(24.0 \mathrm{mg} / \mathrm{mL})$ and the UV-vis and CD spectra were recorded (Figure 4), prior to any thermal treatment. A $\lambda_{\text {max }}$-value of $520 \mathrm{~nm}$ is observed, which is quite common for aggregated HT-P3ATs. There is also a slight indication of the presence of the typical, aggregation-related absorption band around $615 \mathrm{~nm}$. The CD spectrum (Figure 4 , b) clearly consists of a bisignate Cotton effect, due to chiral exciton coupling. In addition, the monosignate Cotton effect, associated with the red-shifted band around $615 \mathrm{~nm}$, is weakly present. 
Next, the polymer film was heated at $150^{\circ} \mathrm{C}$, well above $\mathrm{T}_{\mathrm{m}}$, for several minutes and immediately cooled down in methanol at $-78^{\circ} \mathrm{C}$ (P3-fast). After drying the sample under an air-flow, the UV-vis and CD spectra were measured. Both spectra of the fast-cooled sample are almost identical to those of the spincoated sample, prior to any annealing. If, however, the sample is heated at $150^{\circ} \mathrm{C}$ for several minutes and subsequently slowly cooled down (cooling rate: $\left.-10^{\circ} \mathrm{C} / \mathrm{min}\right)(\mathbf{P 3}$-slow), significant changes are observed in both the UV-vis and CD spectrum. The maximum absorption does not change significantly, but the spectrum shows a more defined fine-structure. At $615 \mathrm{~nm}$, the red-shifted, aggregation-related absorption band becomes more pronounced. Also in the CD spectrum, a monosignate Cotton effect, associated with the aggregation-related absorption band, is more intense. These observations suggest that the fraction of polymer segments, interacting via $\pi$-interactions, is significantly higher. Besides the appearance of the monosignate Cotton effect in the CD spectrum, other remarkable changes are observed: the intensity of the $\mathrm{CD}$ signal has increased significantly and its sign is inverted, a phenomenon already described by Meijer and coworkers. ${ }^{47}$ Upon fast cooling, a metastable, chiral aggregate is formed. This is a kinetically favored process, while upon slow cooling, the thermodynamically most stable form is obtained. In general, the sign of the bisignate Cotton effect is correlated with the handedness of the helical stacking of the polymer chains. Slow cooling results in right-handed helically stacked aggregates while fast cooling results in a left-handed stacking. The magnitude of the CD signal is mainly governed by the distance and the angle between polymer chains. $^{48-50}$

From these experiments, we can conclude that annealing has an important influence on the fraction of aggregates, as illustrated by the presence of the red-shifted, aggregation-related absorption band, and the shape of the aggregates, governing the intensity and the sign of the Cotton effects in the CD spectrum. It is important to note that the $\pi$-interactions themselves do not need to be different. They are essentially governed by the molecular structure, which is the same in all samples. 

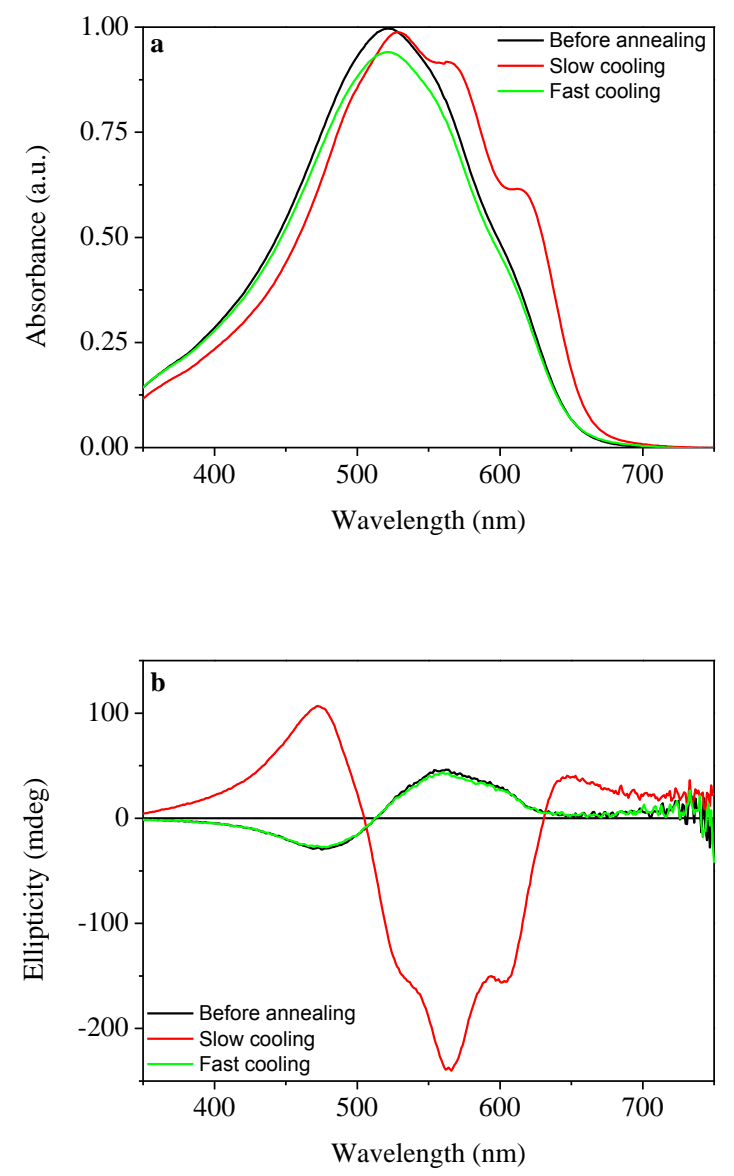

Figure 4. UV-vis (a) and CD spectra (b) of a $\mathbf{P 3}$ film (spincoated from a chloroform solution; 24.0 $\mathrm{mg} / \mathrm{mL} ; 1500 \mathrm{rpm})$ subjected to different annealing procedures.

It must be mentioned that due to their different history spincoated films and powders obtained by precipitation can have a significant different history. However, here we compare a film which was molten (and therefore, its history was erased) and molten powders. In both cases, the history (cooling starting from melt) and the heat treatment (slow or fast cooling) were the same. Therefore, these samples can be compared, provided that any mass-effect is ruled out. This means that the observed effects, visualized by UV-vis and CD spectroscopy, should be independent of the amount of material used. In this respect, films with various thicknesses (P3 spincoated from chloroform solutions; 9.4 $\mathrm{mg} / \mathrm{mL}, 24 \mathrm{mg} / \mathrm{mL}$ and $40 \mathrm{mg} / \mathrm{mL}$ ) were prepared and subjected to the same annealing procedures. Their UV-vis and CD spectra were recorded (see S5). The $\mathrm{g}_{\mathrm{abs}}$-value of HT-P3AT can be considered as an intrinsic material property, and should therefore be independent of the film thickness. ${ }^{51}$ This would 
imply thatannealing has the same effect on all samples, no matter how much material is used. The $g_{a b s}{ }^{-}$ value was calculated (also see S5), showing no effect of the film thickness for the samples before annealing and those subjected to fast cooling. The slow-cooling procedure, however, shows a slight film

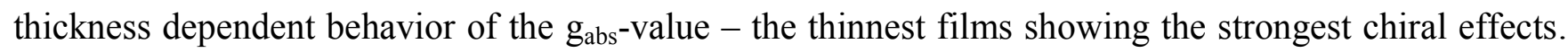
Both observations indicate small differences in the structural parameters of the supramolecular aggregates, depending on the film thickness. Similar results were reported by Joshi et al. ${ }^{52}$, using XRD to demonstrate the influence of the film thickness on the crystalline structure of HT-P3AT. In summary,

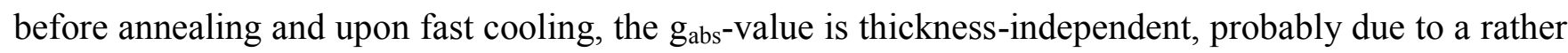
low degree of organization. However, upon slow cooling, the $g_{a b s}$-value is higher and also shows some thickness dependence, probably caused by differences in the structural parameters of the supramolecular $\pi$-stacks. Fortunately, at $\lambda \sim 615 \mathrm{~nm}$, the wavelength of the red-shifted absorption band, the deviation is relatively small. Therefore, the relevant chiroptical properties, measured on spincoated films, can be correlated with the magnetic properties measured for which the thicker, pressed films were used.

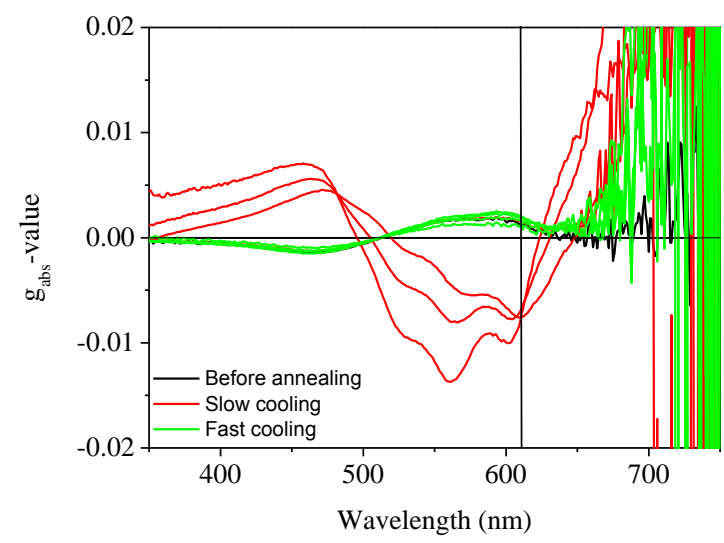

Figure 5. $\mathrm{g}_{\mathrm{abs}}$-value for $\mathbf{P 3}$ films of different thicknesses and subjected to different annealing procedures.

\section{ESR spectroscopy}

The magnetic behavior of all polymer powder samples was initially studied by ESR spectroscopy at $300 \mathrm{~K}$. The samples were treated with an alcoholic hydrazine solution and dried under argon to avoid 
polaron formation by oxidation to ambient air. The inferred spin densities, g-values and line widths are reported in Table 2 .

Table 2. ESR spin densities $\left(S=\frac{1}{2}\right)$, g-value and line widths $\left(\Delta \mathrm{B}_{\mathrm{pp}}\right)$ observed on different samples P14 before and after annealing. S1 and S2 refer to the two different signals observed. All measurements were performed at $300 \mathrm{~K}$.

\begin{tabular}{|c|c|c|c|c|c|c|}
\hline \multirow[b]{2}{*}{ Polymer } & \multicolumn{3}{|c|}{$\mathrm{S} 1$} & \multicolumn{3}{|c|}{$\mathrm{S} 2$} \\
\hline & $\begin{array}{l}\text { Spin Density } \\
\left(10^{16} \text { spins } / g\right)\end{array}$ & $\begin{array}{c}\text { g-value } \\
( \pm 0.0001)\end{array}$ & $\begin{array}{l}\Delta \mathrm{B}_{\mathrm{pp}} \\
(\mathrm{G})\end{array}$ & $\begin{array}{l}\text { Spin Density } \\
\left(10^{16} \text { spins } / g\right)\end{array}$ & $\begin{array}{c}g \text {-value } \\
( \pm 0.0001)\end{array}$ & $\begin{array}{l}\Delta \mathrm{B}_{\mathrm{pp}} \\
(\mathrm{G})\end{array}$ \\
\hline P1 & (1.3 & 2.0037 & 6.9 & - & - & - \\
\hline $\mathbf{P 2}$ & 1.8 & 2.0036 & 5.3 & - & - & - \\
\hline P3 & 0.94 & 2.0042 & 5.4 & 0.27 & 2.0023 & 2.7 \\
\hline P4 & 1.4 & 2.0043 & 5.2 & 1.1 & 2.0023 & 2.8 \\
\hline P3-fast & 0.48 & 2.0045 & 5.3 & 0.18 & 2.0022 & 2.8 \\
\hline P3-slow & 2.1 & 2.0041 & 5.3 & 1.1 & 2.0022 & 1.4 \\
\hline
\end{tabular}

${ }^{\mathrm{a}}$ Accuracy estimated at $\leq 10 \%$

Upon variation of the molar mass (P1-4), clear changes are observed in the spectral line shape. The low molar mass polymers (P1-2) show a single line at zero crossing g-value 2.0036 (spectra: see S6). For higher molar masses (P3-4), two different, overlapping signals, S1 and S2, are present. The lineshape of signal S2 is Lorentzian, while signal S1 could be adequately fitted by a Voigt shape of Lorentzian/Gaussian fraction of 2 and 0.5 for sample P3 and P3-slow, respectively, allowing the determination of the g-values and line widths of both signals (Table 2 and Figure 6). These observations indicated the presence of two types of spin centra which experience a different magnetic environment. In addition, noteworthy is that at higher molar masses the relative intensity of the S2 system increases compared to S1.

The influence of sample annealing on the spin properties was also monitored. P3-fast shows a similar ESR spectrum as P3. Two overlapping signals, corresponding to the presence of two different spin systems, are observed, and the relative intensity of both spectral lines is the same for both samples. On the other hand, P3-slow shows a significant increase in the relative intensity of S2, as shown in Figure 6. Clearly, annealing has an influence on the ratio of S1 and S2. 

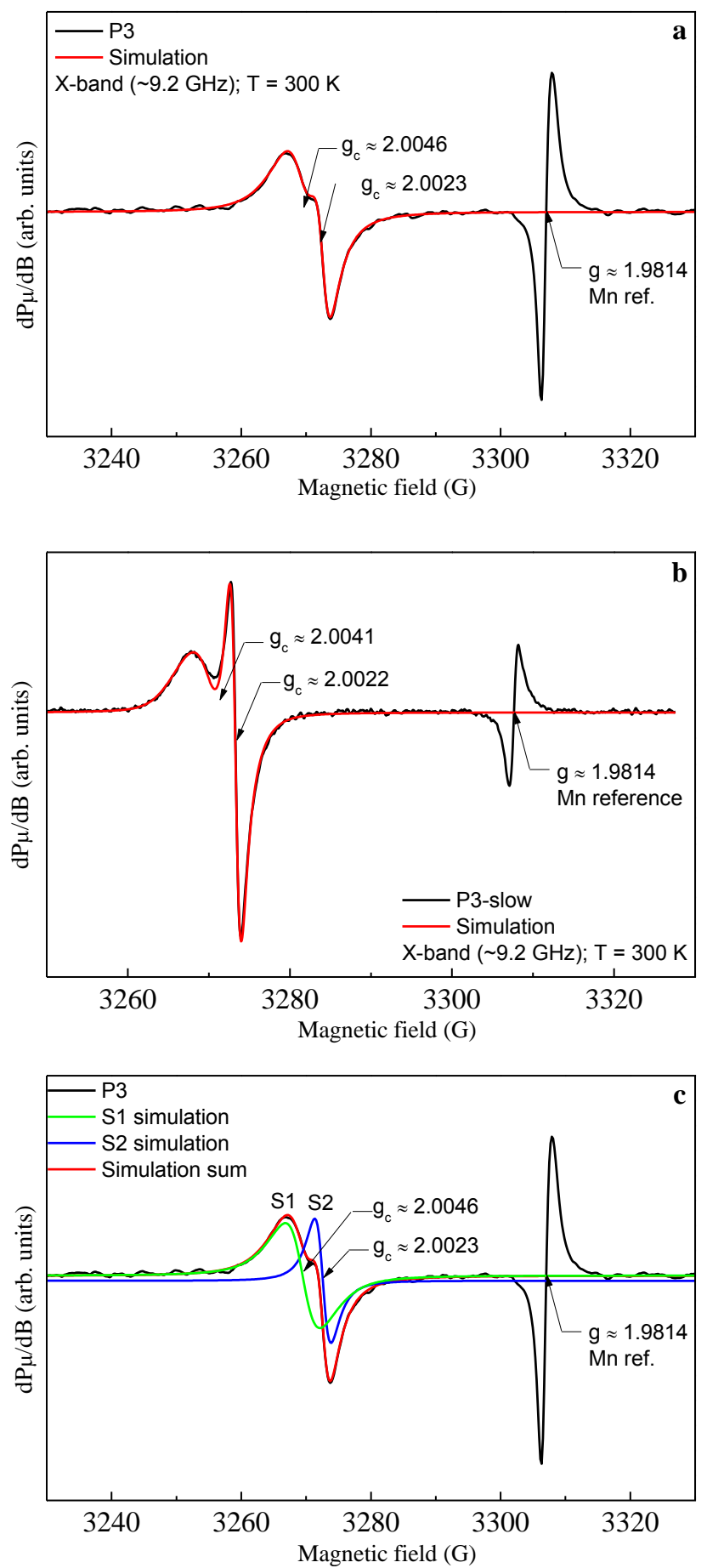

Figure 6. X-band first derivative absorption ESR spectra of (a) P3, (b) P3-slow and (c) simulation of the P3 spectrum, using $P_{\mu}=0.1 \mathrm{~mW}$ and $B_{m}=0.25 \mathrm{G}$. The narrow signal at the right stems from a comounted $\mathrm{MgO}: \mathrm{Mn}^{2+}$ marker signal. The red solid curves represent the optimized simulation results using a Lorentzian shape for signal S2 and a Voigt function of Lorentz/Gauss fraction of 2 and 0.5 for the cases (a) and (b), respectively, leading to the data indicated in Table 2.

As the molecular structure of all samples is the same, the different spin systems S1 and S2 must originate from differences in the supramolecular structure. ${ }^{53-55}$ A powder sample of HT-P3AT typically consists of well-ordered, aggregated polymer chains with unaggregated regions from the chain end 
groups. Both the aggregates and the amorphous regions contain unpaired electrons, which behave differently. In the highly ordered aggregates, the spins (S2) are strongly delocalized. This behavior is confirmed by the g-value, which is close to the free electron g-value. Indeed, the spins do not seem to interact with their environment as this would give rise to a shift of the g-factor. Also any signs of hyperfine interaction is absent, supporting the delocalized nature of S2. In addition, the line widths are significantly more narrow ascribed to the motional narrowing effect. ${ }^{54}$ The first spin system (S1), on the other hand, is located in the more amorphous regions. The spins are less delocalized and deviations from the free electron g-value are observed, ascribed to spin orbit coupling. In addition, the line widths increase as the contribution of the Elliott mechanism, ${ }^{56-57}$ causing line broadening at elevated temperatures, overwhelms the motional narrowing effect.

Increasing the molar mass (P1 to $\mathbf{P 4 )}$ increases the relative intensity of S2 relative to S1. This means that the fraction of highly delocalized spins increases, which is consistent with a larger crystalline fraction upon increasing the molar mass. In this perspective, the behavior of P3-slow is also explained. Upon slow cooling of the polymer melt, the amount of well-organized aggregates increases, which is accompanied by an increasing intensity of the S2 system.

Finally, the temperature dependence of the magnetic susceptibility was studied for both spin systems. As presented in Figure 7, both spin systems show a paramagnetic Curie-Weiss behavior, which confirms the absence of interactions between the unpaired electrons.

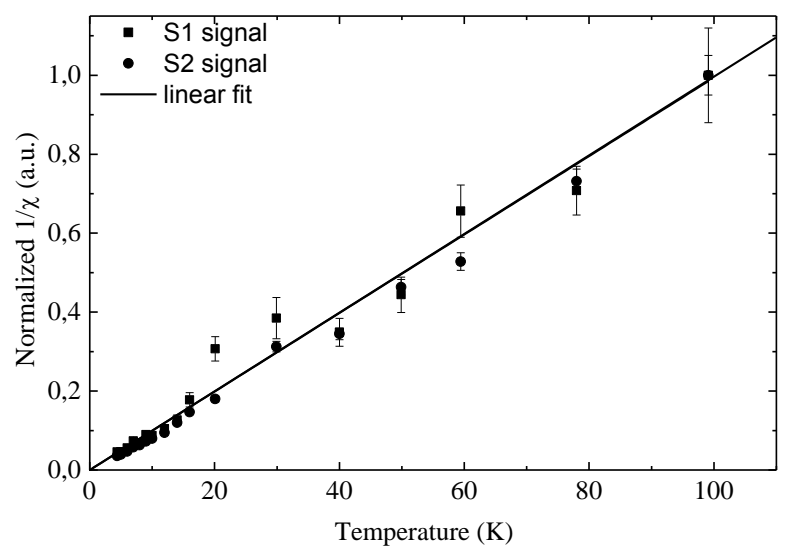

Figure 7. Temperature dependence of the magnetic susceptibility of P3, measured by ESR spectroscopy. Both spin systems S1 and S2 show a paramagnetic Curie-Weiss behavior. 


\section{SQUID magnetometry}

The magnetic behavior of P1-4 was investigated by temperature-dependent SQUID magnetometry. From the magnetization $(\mathrm{M})$ versus the applied magnetic field $(\mathrm{H})$ curves, a diamagnetic contribution, caused by the large number of paired spins and sample holder, was subtracted. All samples show a weak ferromagnetic hysteretic behavior at $5 \mathrm{~K}$ (For P1,3-4, see Figure 8), which is in accordance with the observations made earlier for $\mathbf{P 2}$ (see S7). ${ }^{26}$ The magnitude of this ferromagnetism is characterized by the coercivity and is the same for all samples $(\sim 180 \mathrm{Oe})$. The magnetization at saturation, on the contrary, is clearly influenced by the molar mass of the samples. P4, which has the highest molar mass, shows the highest saturation magnetization, while P1 the lowest. In addition, the $\mathrm{M}(\mathrm{H})$ curves at $300 \mathrm{~K}$ showed a superparamagnetic behaviour. Since the magnitude of the signal at $300 \mathrm{~K}$ equals the uncertainty of the background substraction, no further conclusions could be drawn.

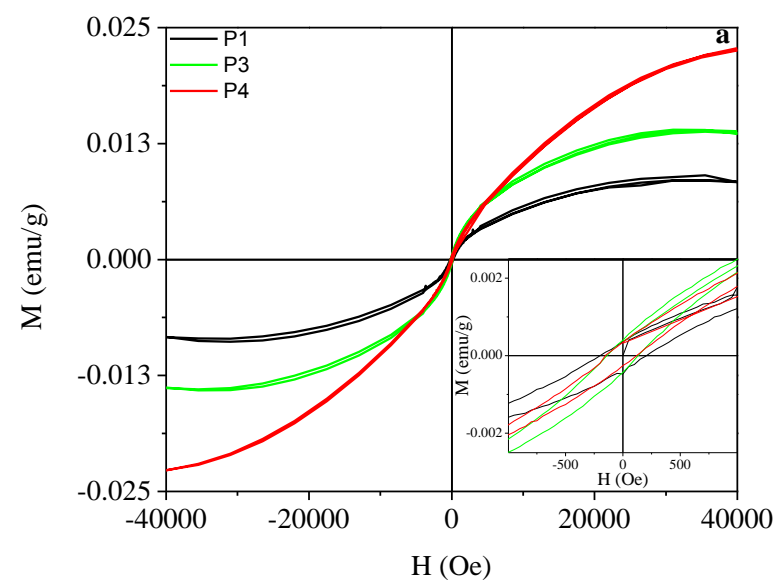

Figure 8. Magnetic hysteresis loops of P1,3-4 at 5 K.

Similar results were obtained upon sample annealing, as depicted in Figure 9. The magnetic behavior was again evaluated at $5 \mathrm{~K}$ and $300 \mathrm{~K}$, showing a ferromagnetic and superparamagnetic behavior, respectively. At low temperature (Figure 9, a), all samples show a similar coercive field ( 180 Oe), but again clearly differ in the value of their saturation magnetization. The procedure in which $\mathbf{P 3}$ was melted and slowly cooled (P3-slow), gives rise to the highest magnetization values, while P3 before 
treatment and after fast cooling (P3-fast) show an almost identical saturation magnetization value. At room temperature (Figure 9, b), a similar trend is observed, i.e. the highest saturation magnetization occurs for P3-slow.
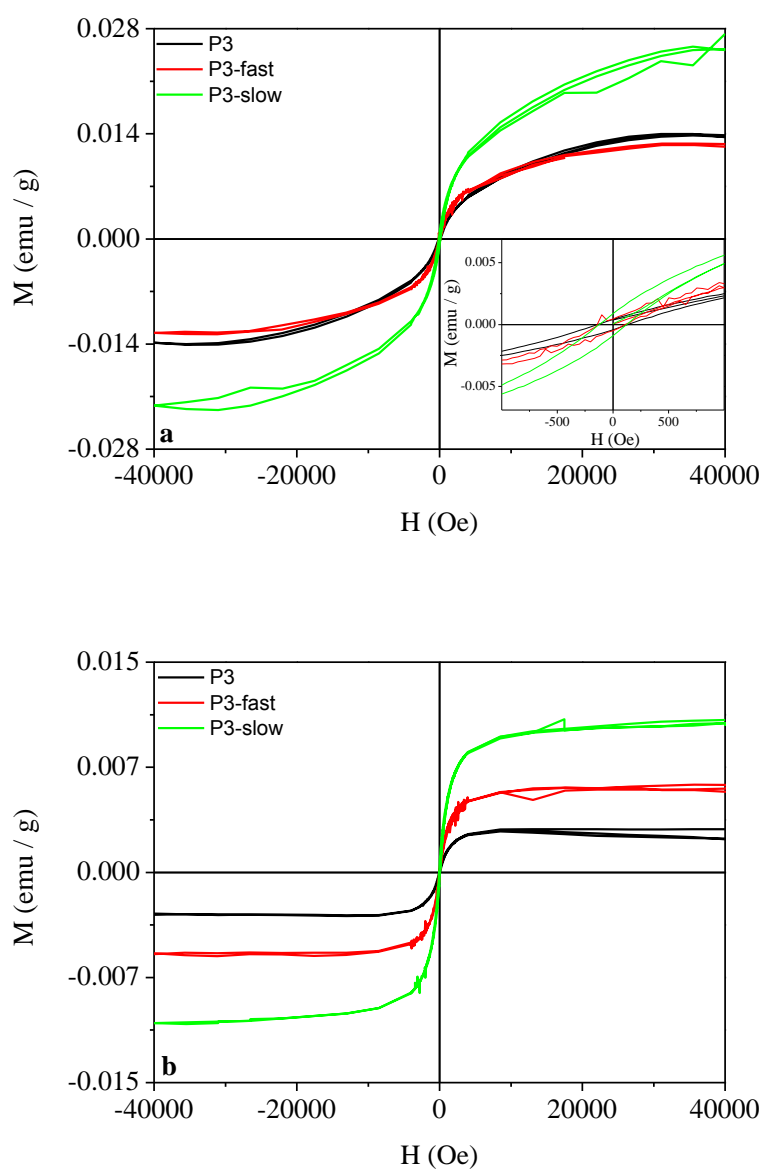

Figure 9. Magnetic hysteresis loops of $\mathbf{P 3}$ subjected to different annealing conditions. (a) $\mathrm{M} / \mathrm{H}$ behavior at $5 \mathrm{~K}$; (b) $\mathrm{M} / \mathrm{H}$ behavior at $300 \mathrm{~K}$.

The results suggest a relationship between the supramolecular organization of HT-P3AT and the magnetic behavior. Usually, magnetism is related to the presence of unpaired electrons. All HT-P3AT samples indeed contain low amounts of spins, even in the neutral state, as was revealed by ESR spectroscopy. However, the number of ESR-active centra does not correspond to the number of Bohr magnetons, derived from the saturation magnetization (SQUID). ${ }^{26}$ As evident from Table 3 , the number of Bohr magnetons, significantly exceeds the ESR values, suggesting the presence of an additional spin system, apparently not resolved by ESR spectroscopy. On the other hand, this spin system gives rise to a small but clearly observable magnetic hysteresis effect at $5 \mathrm{~K}$. These results suggest that this 
contribution originates from another mechanism, such as orbital magnetism, possibly associated with the aromatic repeating units of the conjugated polymer backbone. ${ }^{58-59}$ It must be mentioned that chirality or the helicity of the stacked polymer chains is not required for this contribution, as similar results are obtained in achiral P3ATs, in which the polymer chains stack in a parallel fashion. ${ }^{26}$

Table 3. Comparison of the ESR-derived spin density and the number of Bohr magnetons, determined by SQUID magnetometry.

\begin{tabular}{cccc}
\hline Polymer & $\begin{array}{c}\text { Spin Density } \\
\left(10^{16} \text { spins/g }\right)^{\mathrm{a}}\end{array}$ & $\begin{array}{c}\text { Bohr magnetons } \\
\left(10^{17} / \mathrm{g}\right)^{\mathrm{b}}\end{array}$ & Ratio $^{\mathrm{c}}$ \\
\hline \hline P1 & 1.3 & 3.1 & 0.041 \\
P3 & 1.2 & 3.1 & 0.039 \\
P4 & 2.5 & 1.7 & 0.155 \\
\hline P3-fast & 0.66 & 6.5 & 0.010 \\
P3-slow & 3.2 & 12 & 0.025 \\
\hline
\end{tabular}

${ }^{\mathrm{a}}$ Sum of S1 and S2 signals, determined by ESR at

$300 \mathrm{~K},{ }^{\mathrm{b}}$ determined by SQUID at $300 \mathrm{~K},{ }^{\mathrm{c}}$ Ratio of the spin density and the number of Bohr magnetons.

In contrast, a clear correlation is observed between the number of Bohr magnetons and the strength of the red-shifted absorption band which originates from multiple, strongly interacting polymer chains. Both the molar mass and annealing have a significant influence on the saturation magnetization. As evidenced in Figure 10, an increase in the amount of strongly interacting, planar polymer chains, quantified by taking the relative integration of the red-shifted absorption band, induces higher magnetization values at saturation. However, a similar number of Bohr magnetons are found in poly(3alkoxythiophene)s (P3AOT), ${ }^{26,37,60}$ which do not organize into large aggregates. The typical, red-shifted absorption band is in these materials also absent. As a consequence, the high number of Bohr magnetons in the case of HT-P3AT cannot exclusively be ascribed to aggregation nor to $\pi$-interactions. The observed trend between the saturation magnetization and the aggregation-related absorption band must therefore be a manifestation of some underlying effect. As P3ATs aggregate, the polymer chains are planarized, allowing the $\pi$-systems of different chains to interact. Upon increasing the amount of aggregates in a sample, for instance by changing the molar mass or by annealing, a higher fraction of 
such planar polymer chains is present. This suggests a possible relationship between the fraction of planar polymer chains, found by UV-vis spectroscopy and/or DSC, and the saturation magnetization (Figure 10). In this perspective, our hypothesis explains the high number of Bohr magnetons observed for HT-P3AOT in which S-O-interactions ${ }^{61}$ induce a planar polymer conformation, which is for example also reflected in its large $\lambda_{\max }$ value. In summary, the high number of Bohr magnetons is related to the planarization of the polymer backbone.

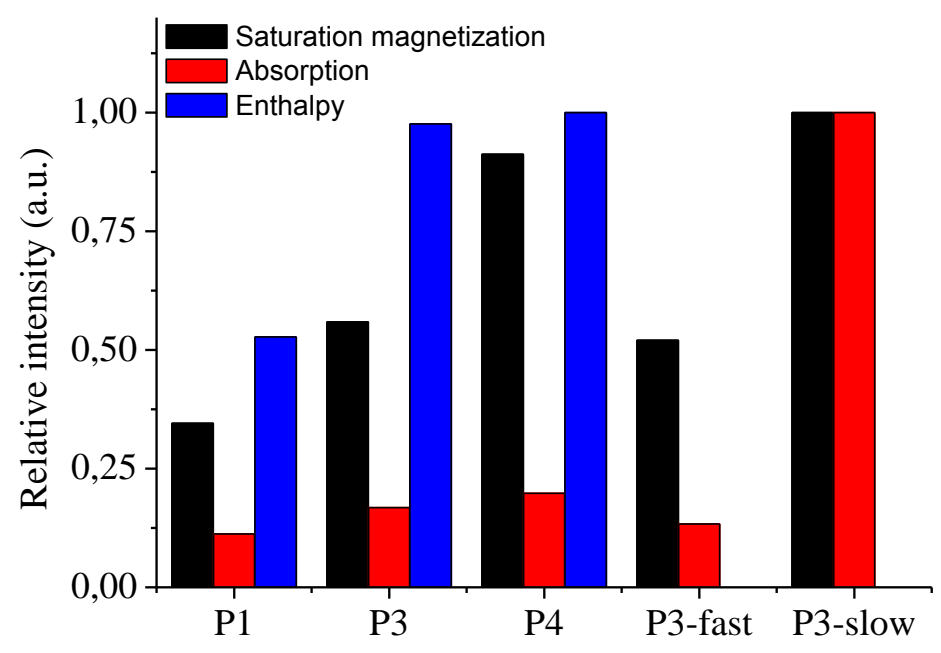

Figure 10. Comparison of the relative intensity of the saturation magnetization determined from SQUID measurements at $5 \mathrm{~K}$, the red-shifted absorption band and the enthalpy of fusion, determined by DSC.

The effects of the supramolecular behavior on the coercivity were also monitored. However, the coercive field is clearly independent of the degree of ordering. In addition, changing the shape of the aggregates by varying the annealing procedure, as nicely illustrated by CD spectroscopy, does not have a distinct effect on the coercive field. Clearly, changes on a supramolecular level are not able to induce deviations of the coercivity. On the other hand, as shown in our previous work, changing the nature of the substituent does result in a variation of the coercivity. For instance, head-to-tail poly(3alkylthiothiophene)s (HT-P3ATTs) have a remarkably higher coercive field compared to HT-P3AT. Clearly, the coercivity is influenced by the molecular structure of the polymer and, closely related to this parameter, by the efficiency in which $\pi$-systems overlap. As all the samples under discussion in this 
article concern HT-P3ATs with the same substituent, which all show a similar $\pi$-interaction, the coercivities can indeed be understood to be the same. .

\section{Conclusion}

In conclusion, samples of HT-P3AT with different molar masses (P1-4) were prepared by fractionation. The chiroptical behavior was evaluated, showing a correlation between the relative intensity of the red-shifted, aggregation-related absorption band and the molar mass. In addition, in the CD spectrum, a monosignate Cotton effect is revealed with this distinct absorption, which can be ascribed to a transition that is delocalized over multiple stacked polymer chains. Also the influence of thermal annealing on the chiroptical behavior of $\mathbf{P 3}$ was evaluated. A different cooling rate clearly has a significant effect on the number and shape of the aggregates, as illustrated by UV-vis and CD spectroscopy.

Powder samples of P1-4 and annealed powder samples (P3-fast/slow) were characterized by ESR spectroscopy and SQUID magnetometry. The ESR spectra of the high molar mass polymers P3-4 consist of two lines, one of Lorentzian shape, the other of Voigt shape. The spectral characteristics (gvalue and line width) indicate the presence of two spin systems, i.e. highly delocalized spins situated in the crystalline domains, giving rise to a sharp signal and more confined spins localized in the amorphous part, corresponding to a broader line shape. The intensity of the signal is influenced by the molar mass and by annealing, as this affects the crystallinity. However, as previously reported, these spin systems are paramagnetic. SQUID magnetometry shows for all samples a ferromagnetic behavior at $5 \mathrm{~K}$ and a superparamagnetic behavior at $300 \mathrm{~K}$. Upon increasing the molar mass and upon slow annealing, the saturation magnetization increases. Remarkably, the coercivity remains constant for all samples. We hypothesize that the magnitude of the magnetization is governed by the fraction of planar polymer chains, while the coercivity seems to depend on the molecular structure, and related to this, the efficiency of $\pi$-interactions between different polymer chains. 
Acknowledgment: We are grateful to the Fund for Scientific Research (FWO-Vlaanderen), the Flemish Concerted Action (GOA), the Belgian Interuniversity Poles of Attraction (IAP), and the Air Force Office of Scientific Research. J.C. are doctoral fellows of the Institute for the Promotion of Innovation through Science and Technology in Flanders (IWT-Vlaanderen).

Supporting Information Available: GPC traces and ${ }^{1} \mathrm{H}$ NMR spectra of the different polymer fractions, the UV-vis spectra of P1-4 in chloroform and the deconvolution of the UV-vis spectra of P1-4 upon aggregation and annealing are given. Also the UV-vis and CD spectra concerning the influence of the film thickness (of P3, upon annealing) on the supramolecular behavior, is given. Finally, the ESR spectra of P1-2,4 and P3-fast are presented and the M (H) behavior of P2 (5 K and $300 \mathrm{~K}$ ) and P1-4 (at $300 \mathrm{~K}$ ) is given. This information is available free of charge via the Internet at http://pubs.acs.org.

\section{References}

(1) Handbook of Conducting Polymer, $3^{\text {rd }}$ Ed., Conjugated Polymers: Theory, Synthesis, Properties and Characterization; $3^{\text {rd }}$ Ed. ed.; Skotheim, T. A.; Reynolds, J. R., Eds.; CRC Press: Boca Raton, 2007.

(2) Brédas, J. L.; Street, G. B. Acc. Chem. Res. 1985, 18, 309.

(3) Fisher, A. J.; Hayes, W.; Wallace, D. S. J. Phys. Condens. Matter 1989, 1, 5567.

(4) Ikoma, T.; Okada, S.; Nakanishi, H.; Akiyama, K.; Tero-Kubota, S.; Mobius, K.; Weber, S. Phys. Rev. B 2002, 66.

(5) Sercheli, M. S.; Walmsley, L.; Rettori, C.; Correa, A. A.; Bulhoes, L. O. S.; Pereira, E. C. Phys. Status Solidi B 2000, 220, 631.

(6) Ovchinnikov, A. A. Theor. Chim. Acta 1978, 47, 297.

(7) Borden, W. T.; Davidson, E. R. J. Am. Chem. Soc. 1977, 99, 4587.

(8) Kothe, G.; Denkel, K. H.; Summerma.W Angew. Chem. Int. Ed. 1970, 9, 906.

(9) Rajca, A.; Rajca, S. J. Chem. Soc., Perkin Trans. 2 1998, 1077. 
(10) Tomioka, H.; Hattori, M.; Hirai, K.; Sato, K.; Shiomi, D.; Takui, T.; Itoh, K. J. Am. Chem. Soc. 1998, $120,1106$.

(11) Rajca, A.; Rajca, S.; Wongsriratanakul, J. J. Am. Chem. Soc. 1999, 121, 6308.

(12) Nishide, H.; Maeda, T.; Oyaizu, K.; Tsuchida, E. J. Org. Chem. 1999, 64, 7129.

(13) Kaneko, T.; Makino, T.; Miyaji, H.; Teraguchi, M.; Aoki, T.; Miyasaka, M.; Nishide, H. J. Am. Chem. Soc. 2003, 125, 3554.

(14) Murata, H.; Miyajima, D.; Nishide, H. Macromolecules 2006, 39, 6331.

(15) Long, Y.; Chen, Z.; Shen, J.; Zhang, Z.; Zhang, L.; Xiao, H.; Wan, M.; Duvail, J. L. J. Phys. Chem. B 2006, 110, 23228.

(16) Zaidi, N. A.; Giblin, S. R.; Terry, I.; Monkman, A. P. Polymer 2004, 45, 5683.

(17) Dallas, P.; Stamopoulos, D.; Boukos, N.; Tzitzios, V.; Niarchos, D.; Petridis, D. Polymer 2007, $48,3162$.

(18) Kahol, P. K.; Raghunathan, A.; McCormick, B. J. Synth. Met. 2004, 140, 261.

(19) Mizoguchi, K.; Kachi, N.; Sakamoto, H.; Kume, K.; Yoshioka, K.; Masubuchi, S.; Kazama, S. Synth. Met. 1997, 84, 695.

(20) Nascimento, O. R.; de Oliveira, A. J. A.; Pereira, E. C.; Correa, A. A.; Walmsley, L. J. Phys. Condens. Matter 2008, 035214 (8 pp.).

(21) de Paula, F. R.; Walmsley, L.; Pereira, E. C.; de Oliveira, A. J. A. J. Magn. Magn. Mater. 2008, 320, E193.

(22) Sugiyama, K.; Kojima, T.; Fukuda, H.; Yashiro, H.; Matsuura, T.; Shimoyama, Y. Thin Solid Films 2008, 516, 2691.

(23) Nalwa, H. S. Phys. Rev. B Condens. Matter 1989, 39, 5964.

(24) Konkin, A.; Roth, H. K.; Scharff, P.; Aganov, A.; Ambacher, O.; Sensfuss, S. Solid State Commun. 2009, 149, 893.

(25) Nechtschein, M.; Devreux, F.; Genoud, F.; Guglielmi, M.; Holczer, K. Phys. Rev. B Condens. Matter 1983, 27, 61. 
(26) Vandeleene, S.; Jivanescu, M.; Stesmans, A.; Cuppens, J.; Van Bael, M. J.; Yamada, H.; Sato, N.; Verbiest, T.; Koeckelberghs, G. Macromolecules 2010, 43, 2910.

(27) Chabinyc, M. L. Polym. Rev. 2008, 48, 463.

(28) Stesmans, A. Phys. Rev. B Condens. Matter 1993, 48, 2418.

(29) Loewe, R. S.; Ewbank, P. C.; Liu, J.; Zhai, L.; McCullough, R. D. Macromolecules 2001, 34, 4324.

(30) Ishikawa, H.; Xu, X. F.; Kobayashi, A.; Satoh, M.; Suzuki, M.; Hasegawa, E. J. Phys. D: Appl. Phys. 1992, 25, 897.

(31) Ishikawa, H.; Amano, K.; Kobayashi, A.; Satoh, M.; Hasegawa, E. Synth. Met. 1994, 64, 49.

(32) Hourquebie, P.; Olmedo, L. Synth. Met. 1994, 65, 19.

(33) Trznadel, M.; Pron, A.; Zagorska, M. Macromolecules 1998, 31, 5051.

(34) Zahn, S.; Swager, T. M. Angew. Chem. Int. Ed. 2002, 41, 4225.

(35) Babudri, F.; Colangiuli, D.; Di Bari, L.; Farinola, G. M.; Omar, O. H.; Naso, F.; Pescitelli, G. Macromolecules 2006, 39, 5206.

(36) Brown, P. J.; Thomas, D. S.; Kohler, A.; Wilson, J. S.; Kim, J. S.; Ramsdale, C. M.; Sirringhaus, H.; Friend, R. H. Phys. Rev. B 2003, 67.

(37) Vangheluwe, M.; Verbiest, T.; Koeckelberghs, G. Macromolecules 2008, 41, 1041.

(38) Vandeleene, S.; Van den Bergh, K.; Verbiest, T.; Koeckelberghs, G. Macromolecules 2008, 41, 5123.

(39) Bilo, E. J. Excel for Chemists; $2^{\text {nd }}$ ed., 2001.

(40) Zen, A.; Saphiannikova, M.; Neher, D.; Grenzer, J.; Grigorian, S.; Pietsch, U.; Asawapirom, U.; Janietz, S.; Scherf, U.; Lieberwirth, I.; Wegner, G. Macromolecules 2006, 39, 2162.

(41) Liu, J. H.; Arif, M.; Zou, J. H.; Khondaker, S. I.; Zhai, L. Macromolecules 2009, 42, 9390.

(42) Wu, Z. Y.; Petzold, A.; Henze, T.; Thurn-Albrecht, T.; Lohwasser, R. H.; Sommer, M.; Thelakkat, M. Macromolecules 2010, 43, 4646. 
(43) Pascui, O. F.; Lohwasser, R.; Sommer, M.; Thelakkat, M.; Thurn-Albrecht, T.; Saalwachter, K. Macromolecules 2010, 43, 9401.

(44) Virkar, A. A.; Mannsfeld, S.; Bao, Z. A.; Stingelin, N. Adv. Mater. 2010, 22, 3857.

(45) Brinkmann, M.; Rannou, P. Macromolecules 2009, 42, 1125.

(46) Muller, C.; Zhigadlo, N. D.; Kumar, A.; Baklar, M. A.; Karpinski, J.; Smith, P.; Kreouzis, T.; Stingelin, N. Macromolecules 2011, 44, 1221.

(47) Bouman, M. M.; Meijer, E. W. Adv. Mater. 1995, 7, 385.

(48) Rodger, A.; Nordén, B. Circular Dichroism and Linear Dichroism Oxford, 1997.

(49) Cornelis, D.; Peeters, H.; Zrig, S.; Andrioletti, B.; Rose, E.; Verbiest, T.; Koeckelberghs, G. Chem. Mater. 2008, 20, 2133.

(50) Purdie, N.; Brittain, H. G. Analytical Applications of Circular Dichroism Amsterdam, 1994.

(51) Lakhwani, G.; Koeckelberghs, G.; Meskers, S. C. J.; Janssen, R. A. J. Chem. Phys. Lett. 2007, 437, 193.

(52) Joshi, S.; Grigorian, S.; Pietsch, U. Phys. Status Solidi A 2008, 205, 488.

(53) Sitaram, V.; Sharma, A.; Bhat, S. V.; Mizoguchi, K.; Menon, R. Phys. Rev. B 2005, 72.

(54) Kanemoto, K.; Kato, T.; Aso, Y.; Otsubo, T. Phys. Rev. B 2003, 68.

(55) Kanemoto, K.; Furukawa, K.; Negishi, N.; Aso, Y.; Otsubo, T. Phys. Rev. B 2007, 76.

(56) Elliott, R. J. Phys. Rev. 1954, 96, 266.

(57) Mizoguchi, K.; Honda, M.; Kachi, N.; Shimizu, F.; Sakamoto, H.; Kume, K. Solid State Commun. 1995, 96, 333.

(58) Himmerich, M.; van Dongen, P. G. J.; Noack, R. M. Eur. Phys. J. B 2006, 51, 5.

(59) O'Hare, A.; Kusmartsev, F. V.; Kugel, K. I.; Laad, M. S. Phys. Rev. B 2007, 76.

(60) Koeckelberghs, G.; Vangheluwe, M.; Samyn, C.; Persoons, A.; Verbiest, T. Macromolecules $\mathbf{2 0 0 5}, 38,5554$.

(61) Villa, E.; Agosti, E.; Castiglioni, C.; Gallazzi, M. C.; Zerbi, G. J. Chem. Phys. 1996, 105, 9461. 


\section{For Table of Contents use only}

Influence of the Supramolecular Organization on the Magnetic Properties of Poly(3-alkylthiophene)s in Their Neutral State.

Steven Vandeleene, Mihaela Jivanescu, André Stesmans, Jo Cuppens, Margriet J. Van Bael, Thierry Verbiest, and Guy Koeckelberghs*

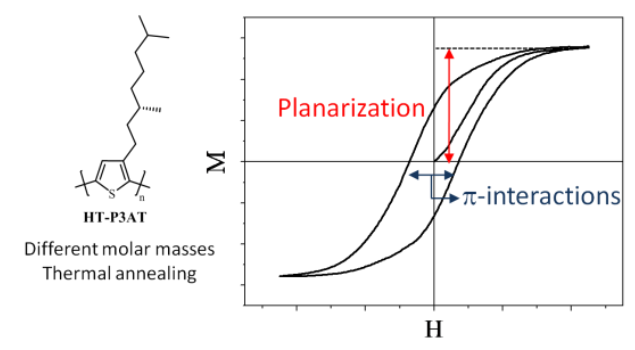

